\title{
Estudios histopatológicos y seguimiento clínico de sarcomas felinos asociados a sitios de inoculación
}

\author{
Histopathological studies and clinical follow-up of feline injection-site \\ sarcomas
}

\author{
Santelices Iglesias Olga Andrea ${ }^{1^{*}}$ ๑ , Wright Carolina ${ }^{1}$, Duchene Adriana \\ Graciela $^{2}$, Risso Miguel Atilio ${ }^{3}$, Risso Paula ${ }^{3}$ () , ZanuzZi Carolina

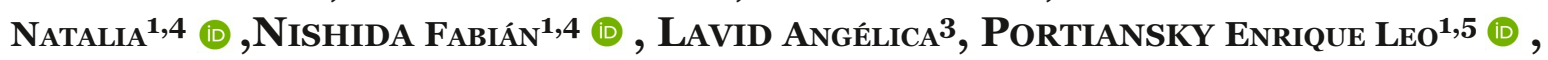 \\ Gimeno Eduardo Juan ${ }^{1,5}$, Barbeito Claudio Gustavo ${ }^{4,5}$
}

1. Laboratorio de Análisis de Imágenes, Facultad de Ciencias Veterinarias (FCV), Universidad Nacional de La Plata (UNLP). 2. Hospital Escuela, FCV, Universidad Nacional de Buenos Aires. 3. Departamento de Epizootiología y Salud Pública, FCV, UNLP. 4. Consejo Nacional de Investigaciones Científicas y Técnicas (CONICET). 5. Laboratorio de Histología Descriptiva Experimental y Comparada y Embriología, FCV, UNLP. Argentina

* Correo electrónico de la autora de contacto: asantelices@fcv.unlp.edu.ar

\begin{abstract}
Resumen
Los sarcomas asociados a sitios de inoculación son neoplasias de origen mesenquimático que aparecen en las regiones corporales utilizadas rutinariamente para la aplicación de vacunas u otros inóculos. Con el objetivo de caracterizar estas neoplasias se seleccionaron 117 tumores diagnosticados como sarcomas posinoculación. Se realizaron estudios histopatológicos e inmunohistoquímicos que permitieron reclasificar los diferentes sarcomas hallados y establecer el porcentaje de células proliferantes. Además, se consideraron los datos demográficos y se realizaron encuestas a los médicos veterinarios para conocer la evolución de los pacientes. El diagnóstico más frecuente fue el de fibrosarcoma (87,18\%). También, se encontraron, en menor proporción, otros tumores como: sarcomas pleomórficos (HFM), osteosarcomas extraesqueléticos y mixosarcomas. El grado histológico más frecuente fue el II y la mayoría presentó abundante inflamación. El promedio de células proliferantes alcanzó el $50,12 \%$. La edad de los pacientes al momento del diagnóstico fue mayor que la informada por otros autores. Si bien los sarcomas asociados a sitios de inoculación en la República Argentina comparten características histopatológicas y clínicas con aquellos informados en otros países, existen diferencias vinculadas a la edad al momento del diagnóstico, a la presentación de las recidivas y al tiempo de sobrevida, entre otras características.
\end{abstract}

\section{Palabras clave}

Histopatología, inmunohistoquímica, seguimiento clínico, sarcomas, sitios de inoculación

\begin{abstract}
Feline injection-site sarcomas are neoplasms of mesenchymal origin frequently found in sites of the body routinely used for inoculation of vaccines and other drugs. With the aim of characterizing these neoplasms, 117 post-inoculation sarcomas were selected and analyzed. Histopathological and immunohistochemical techniques were performed to reclassify different sarcomas and determine cellular proliferation percentages. In addition, demographic data were considered, and surveys were conducted to obtain information about the clinical follow-up of patients. Of all tumors analyzed, fibrosarcoma was the most frequently diagnosed (87.18\%), followed by pleomorphic sarcomas (HFM), extra skeletal osteosarcomas and mixosarcomas. The most frequent histological grade was type II which was associated with great inflammation. Cell proliferation percentage of these tumors was 50.12\%. The average age of diagnosis for these sarcomas was higher than that reported by other authors. Although in Argentina injection-site sarcomas shared histopathological and clinical characteristics with those reported in other countries, differences such as the age in the moment of diagnosis, recurrence and survival were found.
\end{abstract}

\section{Key words}

Histopathology, immunohistochemistry, clinical follow-up, sarcomas, inoculation sites

Fecha de recepción: 10/07/2019

Fecha de revisión: 17/09/2019

Fecha de aprobación: 07/10/2019
ANALECTA VeT 2019; Julio-Diciembre; 39(2):15-26

Impresa ISSN 03655 14-8 Electrónica ISSN 1514-2590

doi.org/10.24215/15142590eo39 


\section{Introducción}

Los sarcomas asociados a sitios de inoculación (SSI) son neoplasias de origen mesenquimático que aparecen en las regiones corporales utilizadas rutinariamente para la aplicación de vacunas u otros inóculos (Shaw et al., 2009). Fueron descriptos por primera vez en 1991, en Estados Unidos de Norteamérica (Hendrick y Goldschmidt, 1991). Posteriormente, se diagnosticaron en la mayoría de los continentes (Burton \& Mason, 1997; Chang et al., 2006; Hendrick \& Brooks., 1994; Nieto et al., 2003). El tipo más frecuente de SSI es un fibrosarcoma sumamente agresivo y recidivante con un alto índice de mortalidad (Goldschmidt \& Hendrick, 2002; Hendrick et al., 1992; Wilcock et al., 2012). Otras variantes, tales como el sarcoma pleomórfico (Hendrick, 2017) o histiocitoma fibroso maligno (HFM), el osteosarcoma, el condrosarcoma y el rabdomiosarcoma, se presentan con menor frecuencia (Dubielzig et al., 1993; Goldschmidt \& Hendrick, 2002; Hendrick et al., 1992; Hendrick \& Brooks, 1994; Wilcock et al., 2012).

La relación entre el desarrollo de sarcomas felinos y la vacunación se estableció a partir de trabajos epidemiológicos. En EE.UU. se asoció al incremento en la vacunación antirrábica de la población felina tras la sanción de una ley de vacunación obligatoria contra la rabia, en 1987 (Deim et al., 2008; Hendrick et al., 1992). El análisis de estos resultados permitió establecer la relación etiológica entre la vacunación y el desarrollo de fibrosarcomas (Hendrick et al., 1992).

\section{Aspectos morfológicos}

Macroscópicamente, los SSI típicos son masas blanquecinas, firmes, bien delimitadas, con un centro cavitado que contiene líquido mucinoso o acuoso (Goldschmidt \& Hendrick, 2002; Hendrick et al., 1992). Es frecuente la presencia de una intensa respuesta inflamatoria periférica, en la que predominan linfocitos y macrófagos (Couto et al., 2002; Goldschmidt \& Hendrick, 2002; Hendrick \& Brooks, 1994). Muchos casos presentan células gigantes intratumorales (Madewell et al., 2001).

\section{Diagnóstico}

La observación microscópica a partir de la biopsia escisional es el método de elección para el diagnóstico (Goldschmidt \& Hendrick, 2002). El diagnóstico de SSI se basa en la identificación de sus características histológicas y en su localización en sitios tradicionalmente usados para la inoculación de vacunas (Wilcock et al., 2012).

\section{Hipótesis respecto a su etiopatogenia}

Los compuestos con aluminio son adyuvantes utilizados comúnmente en vacunas a virus muerto (Deim et al., 2008; Hendrick et al., 1992), incluyendo aquellas que se aplican para prevenir la rabia y la leucemia felina. La presencia de agregados peritumorales de macrófagos con aluminio sustenta el diagnóstico de SSI (Goldschmidt \& Hendrick, 2002; Hendrick et al., 1992). Se postuló que el aluminio generaría una respuesta inflamatoria persistente que induciría la síntesis de factores de crecimiento y citoquinas que estimularían la proliferación de fibroblastos y miofibroblastos. Sin embargo, en un estudio de riesgo, la aparición de SSI también se relacionó con la inoculación de vacunas con adyuvantes que carecían de aluminio y sin adyuvante (Deim et al., 2008; Kass et al., 1993). De todas maneras, estas neoplasias siempre se relacionaron con procesos inflamatorios crónicos. En los felinos, la inflamación persistente como respuesta a la inyección predispondría al desarrollo de una actividad reparativa que, en algunos casos, daría lugar a la formación de una neoplasia (Hendrick et al., 1992; Kass et al., 1993; Woodward, 2011). La magnitud y duración de la inflamación estaría influenciada por la formulación de la vacuna y condicionada por el genoma del paciente (Wilcock et al., 2012).

La inflamación crónica puede inducir la producción de radicales libres y metabolitos que producen daños en el ADN y mutaciones, actuando como iniciador de la carcinogénesis. El ambiente provisto por la inflamación crónica sumado a una predisposición genética alteraría la susceptibilidad ante injurias carcinogénicas (Nieto et al., 2003; O’ Byrne \& Dalgleish, 2001).

\section{Comportamiento biológico y clínico}

Los SSI se originan en sitios de inoculación tales como cuello, región interescapular, parrilla costal, región dorsolumbar, flanco y miembros posteriores (Goldschmidt \& Hendrick, 2002; Hendrick et al., 1992; Hendrick \& Brooks, 1994). A pesar de las diferencias de diagnósticos histopatológicos asociados a los sitios de inoculación, ninguno de ellos brinda diferencias pronósticas para la vida del paciente (Wilcock et al., 2012).

Este tipo de neoplasias no presenta predilección sexual. La edad promedio documentada para su aparición es de 8,1 años, que es menor a la de los sarcomas no asociados a sitios de vacunación que aparecen a una edad promedio de 9,2 años (Goldschmidt \& Hendrick, 2002; Hendrick et al., 1994). Además, los sarcomas no asociados a la vacunación son menos invasivos y recidivantes (Deim et al., 2008).

Se calculó que la prevalencia de los SSI es de 1:1000 a 1:10000 gatos vacunados (Goldschmidt \& Hendrick, 2002; Kass et al., 1993; Wilcock et al., 2012). El intervalo más frecuente entre la vacunación y la aparición del tumor se encuentra entre los 3 meses y los 3 años posinoculación (Hendrick et al., 1992). Sin embargo, puede llegar a los 15 años (Wilcock et al., 2012). 
Los SSI son neoplasias sumamente invasivas, el riesgo de recidiva es alto y pueden ser extirpadas quirúrgicamente de una a tres veces en un lapso de uno a dos años (Goldschmidt \& Hendrick, 2002; Hendrick et al., 1994). Sin embargo, una escisión quirúrgica agresiva, que permita lograr un amplio margen, puede extender el intervalo libre de tumor y la sobrevida (Davidson et al., 1997; Goldschmidt \& Hendrick, 2002; Hershey et al., 2000; Wilcock et al., 2012). Algunos trabajos preliminares sugieren que este intervalo también podría extenderse con el uso de quimioterapia o de combinaciones de inmunoestimulantes y radioterapia (Goldschmidt \& Hendrick, 2002).

En la mayoría de los casos, los gatos son sometidos a eutanasia luego de varias cirugías con o sin terapia adyuvante. El potencial metastásico de estos tumores inicialmente es bajo, pero aumenta con la sobrevida prolongada (Goldschmidt \& Hendrick, 2002; Wilcock et al., 2012). Las metástasis se encontraron en linfonodos regionales, mediastino, pulmones (Briscoe et al., 1998; Goldschmidt \& Hendrick, 2002; Hendrick et al., 1994; Rudmann et al., 1996), hígado y omento (Sandler et al., 1997).

\section{Situación actual}

Luego de haber sido descritos por primera vez en 1991, la identificación de estos sarcomas motivó cambios en los protocolos de vacunación. Así, hacia 1996 se incluyó la recomendación de modificar los sitios de inoculación y, además, se diseñaron vacunas antirrábicas sin adyuvante, para uso en gatos. Sin embargo, no hubo, tras estas modificaciones, cambios ni en la prevalencia ni en la edad de presentación de los SSI. Sí, en cambio, se registró una variación en la ubicación corporal, en correspondencia a los nuevos sitios recomendados para vacunación. Antes de 1996, la localización interescapular era cinco veces más frecuente que las otras. Luego de ese año, el sitio más frecuente se localizó en los miembros posteriores y sus áreas de adyacencia (Shaw et al., 2009; Wilcock et al., 2012). Por estos motivos, no existiría razón para modificar los sitios de inoculación, más allá de la factibilidad de lograr un amplio margen quirúrgico como sucede en el caso de los miembros o del rabo.

Las vacunas sin adyuvante (recombinantes) no causan inflamación significativa. Por lo tanto, no deberían inducir la formación de sarcomas. La falla en la disminución de la prevalencia podría deberse a que, en realidad y pese a las indicaciones, éstas no reemplazaron, en la práctica, a las vacunas tradicionales, debido a su mayor costo (Wilcock et al., 2012).

Algunos autores sostienen que otros productos inoculados pueden promover el desarrollo de sarcomas (MacEwen et al., 2001; Martano et al., 2011). En realidad, cualquier sustancia que genere una respuesta infamatoria local persistente podría llevar al desarrollo de sarcomas en gatos susceptibles. Sin embargo, el incremento de la frecuencia de inoculación de la población felina sería la responsable de la mayor correlación con la enfermedad (MacEwen et al., 2001).

Los objetivos del presente trabajo fueron caracterizar desde el punto de vista histopatológico e inmunohistoquímico a los SSI, establecer su grado de anaplasia (GA) y de inflamación (GI), calcular el porcentaje de células proliferantes (PCP) y detallar la información obtenida en relación con el seguimiento clínico.

\section{Materiales y métodos}

\section{Muestras}

Se seleccionaron casos de archivo (tacos en parafina) de un laboratorio privado de histopatología que recibe muestras de todo el país (Laboratorio Duchene). Se realizó un muestreo consecutivo (Robledo Martín, 2005), tomando todos los casos que cumplieron con los criterios de inclusión y exclusión durante los años 2011 y 2012. Dichos criterios fueron:

1) Criterio de inclusión: se seleccionaron para su estudio todos los tumores diagnosticados como sarcomas posinoculación, en el período antes mencionado, quedando incluidos 185 casos.

2) Criterio de exclusión: los casos con características histológicas típicas, pero localización atípica o desconocida fueron excluidos. La muestra quedó finalmente conformada por 117 casos.

\section{Estudios histopatológicos}

Los cortes histológicos ( $5 \mu \mathrm{m})$, obtenidos a partir de las muestras seleccionadas, fueron coloreados con Hematoxilina y Eosina. Posteriormente se examinaron con un microscopio óptico (CX31, Olympus, Japón).

Los sarcomas seleccionados fueron reclasificados siguiendo los criterios de la OMS (Organización Mundial de la Salud) para tumores mesenquimáticos de la piel y tejidos blandos (Hendrick et al., 1998). Se consideraron como fibrosarcomas aquellos tumores compuestos por haces o fascículos entrelazados de células fusiformes pleomórficas. En cambio, los que presentaron células de fusiformes a estrelladas, de disposición laxa, inmersas en una matriz mucinosa (que adopta una tonalidad celeste con hematoxilina eosina), fueron clasificados como mixosarcomas. Las neoplasias tradicionalmente llamadas histiocitomas fibrosos malignos, fueron denominadas sarcomas pleomórficos (Hendrick, 2017); para su clasificación, se tuvo en cuenta la presencia de abundantes células gigantes multinucleadas, células fusiformes 
y células histiocitoides atípicas. Aquellos que presentaron células pleomórficas con abundante citoplasma eosinofílico, células multinucleadas y células en raqueta (con prolongaciones citoplasmáticas) se clasificaron como rabdomiosarcomas.

Dado que la OMS (Hendrick et al., 1998) no considera dentro de las neoplasias de tejidos blandos a aquellas que forman matriz condroide $u$ osteoide, se recurrió a otros criterios presentes en la bibliografía, tanto de medicina humana (Sangüesa Nebot et al., 2007) como veterinaria (Thompson y Dittmer, 2017), para su clasificación. Por ello, se consideraron osteosarcomas extraesqueléticos a aquellos que forman matriz osteoide, aunque puedan también formar matriz condroide, (Thompson y Pool, 2002). Debido a que existen comunicaciones científicas que mencionan la posibilidad de ocurrencia de metaplasia osteoide y condroide en neoplasias de tejidos blandos (Thompson y Dittmer, 2017), se consideraron criterios adicionales para diferenciar fibrosarcomas con diferenciación condroide y osteoide de osteosarcomas extraesqueléticos. Para ello, se tuvo en cuenta la extensión y diferenciación del tejido osteoide y condroide. Aquellos con un patrón predominante de haces entrelazados de células fusiformes pleomórficas y que, además, presentaron tejido condroide y osteoide, o solo osteoide, diferenciado y en menos del $50 \%$ de la extensión de la muestra, se consideraron fibrosarcomas con metaplasia ósea. En cambio, las neoplasias que presentaron tejido osteoide con diferenciación moderada a pobre, y que se extiende en más del $50 \%$ de la muestra, se consideraron osteosarcomas extraesqueléticos.

El GA se determinó utilizando un esquema propuesto por Couto (Couto et al., 2002), que toma en cuenta la diferenciación celular, el índice mitótico (IM) y el grado de necrosis. De la combinación de dichas características surge la puntuación que permite clasificar las neoplasias en tres grados.

Para establecer el puntaje correspondiente al grado de diferenciación celular se consideraron los siguientes criterios: se asignó un punto a los tumores cuyas células eran similares a las diferenciadas del tejido normal, dos puntos si sus células presentaban fenotipo histológico definido, y finalmente, se asignó tres puntos a aquellos con células pobremente diferenciadas (Couto et al., 2002).

El IM se estableció obteniendo el promedio de mitosis en diez campos, utilizando un objetivo de alta magnificación (PLAN C 40x 0.65 NA, FN 22 y ocular 10X FN 20). Se excluyeron sectores con extensas áreas de inflamación y necrosis. El puntaje que se atribuyó a los sarcomas respetó el siguiente esquema: un punto para IM entre 1 y 9, dos puntos para IM entre 10 y 19 , y tres puntos para IM $\geq 20$ (Couto et al., 2002).

En cuanto al grado de necrosis de los tumores analizados, se computó un punto cuando se verificó su ausencia, dos puntos cuando la necrosis representó menos del 50\% del área de la muestra, y tres puntos cuando fue mayor al 50\% de su área (Couto et al., 2002).

La suma de los puntajes obtenidos para cada criterio analizado constituyó el puntaje final, en función del cual se atribuyó a cada neoplasia uno de los tres grados de anaplasia considerados (Couto et al., 2002).

El GI se estableció al evaluar el infiltrado linfoide peritumoral a baja magnificación (4X) según criterios adaptados a partir de los utilizados por Couto (2002): $\mathrm{O}=$ ausencia de infiltrado linfoide peritumoral, $1=$ escaso infiltrado linfoide (requiere búsqueda cuidadosa), $2=$ infiltrado difuso 0 pequeños agregados en menos del $50 \%$ de los campos, y 3 =agregados linfoides en el $50 \%$ de los campos o más. En este caso, se excluyó uno de los tacos de la población seleccionada, ya que por ser una muestra mínima (biopsia incisional) el área peritumoral, en la que se centra la evaluación, no resultaba representativa.

\section{Estudios inmunohistoquímicos}

Se realizaron estudios inmunohistoquímicos para confirmar la estirpe celular que originó las neoplasias. Para ello se utilizó, como anticuerpos primarios, pancitoqueratina (anti-cytokeratin, Dako, Carpinteria, CA, USA) y vimentina (Vimentin RTU-VIM-V9, Leica Biosystems, Newcastle Ldt, United Kingdom). Además, se seleccionaron 10 sarcomas al azar a los que se les realizó inmunomarcación con anticuerpo anti-desmina (Desmin RTU-DES-DERII, Leica Biosystems, Newcastle Ldt, United Kingdom). Aquellos casos que lo requirieron fueron sometidos a otras pruebas inmunohistoquímicas para confirmar su diagnóstico histopatológico. Los anticuerpos utilizados en estos casos fueron anti-desmina y anti- $\alpha-$ actina (anti-a-actina, [ab7817] Abcam, Cambridge, England) de músculo liso.

También, se realizó la inmunomarcación con anticuerpo anti-antígeno nuclear de proliferación celular (PCNA, clone PC 10, Sigma Chemical Co., St. Louis, MO, EE. UU.) para establecer el PCP. Para ello, se seleccionó una muestra de 54 casos tomados al azar.

Para la técnica inmunohistoquímica se realizaron cortes de $3 \mu \mathrm{m}$ de espesor que se montaron sobre vidrios positivados (HistoSystem, Rosario, Argentina). Estos se desparafinaron, rehidrataron e incubaron con $3 \%$ de $\mathrm{H}_{2} \mathrm{O} 2$ diluida en metanol, para inhibir la peroxidasa endógena, durante 30 minutos a temperatura ambiente, y se lavaron con solución tamponada de fosfato (PBS, $\mathrm{pH}$ 7,4). La recuperación antigénica se realizó en microondas a $800 \mathrm{~W}$, durante 3 minutos en solución tamponada de citrato, $\mathrm{pH}$ 6,0. Los sitios de unión inespecíficos se bloquearon al incubar los cortes con $1 \%$ de albúmina sérica bovina (BSA) diluida en PBS durante 30 minutos a temperatura ambiente, en cámara húmeda. La incubación con 
anticuerpos primarios policlonales (pancitoqueratina dilución 1:500) y monoclonales (anti- $\alpha$-actina, dilución 1:200; PCNA, dilución 1:3000; antivimentina; anti-desmina) se realizó en cámara húmeda durante una noche a $4{ }^{\circ} \mathrm{C}$, la que fue seguida por la incubación con el anticuerpo secundario anti-conejo (EnVision HRP ®, DakoCytomation, Carpinteria, CA, USA) para los anticuerpos policlonales, y anti-ratón (EnVision HRP @, DakoCytomation, Carpinteria, CA, USA) para los anticuerpos monoclonales en cámara húmeda y a temperatura ambiente, durante 30 minutos. Como control negativo de anticuerpo se reemplazó el anticuerpo por $\mathrm{PBS}, \mathrm{pH}$ 7,4. Se utilizaron muestras de intestino delgado como controles negativos y positivos para cada anticuerpo, considerando su especificidad para los tejidos epitelial de revestimiento y glandular, conectivo y muscular liso. Además, se consideraron como controles negativos a los tejidos no neoplásicos de cada muestra. Se utilizó 3,3-diaminobenzidine tetrahydrochloride (DAB) como cromógeno (DakoCytomation, Glostrup, Denmark), y hematoxilina como colorante de contraste.

El PCP se evaluó mediante la inmunomarcación con anticuerpo anti-PCNA, estimando el porcentaje de células con marcación nuclear positiva, al contar estas sobre imágenes digitales tomadas en diez campos obtenidos mediante un objetivo de 40X de magnificación. Las imágenes se capturaron mediante una cámara de video digital (Sony DXC390) montada sobre un microscopio trinocular (CX31, Olympus, Japón) y conectada a una computadora que cuenta con un programa informático de análisis de imágenes digitales (ImagePro Plus v6.3 - Media Cybernetics, USA).

\section{Seguimiento clínico}

Las unidades de observación utilizadas corresponden a los Médicos Veterinarios remitentes y protocolos de remisión de muestras para obtener información de la historia clínica y evolución.

Las variables consideradas fueron: 1) parámetros demográficos: raza, y sexo; 2) parámetros clínicos: edad al momento del diagnóstico, ocurrencia de recidivas, tiempo promedio entre recidivas y sobrevida. Además, se consideró la ubicación anatómica de las neoplasias.

Las técnicas de recolección de la información fueron las siguientes: los parámetros demográficos y la ubicación anatómica se extrajeron de la información enviada por los profesionales remitentes junto con la muestra (protocolos de remisión de muestra). Para la recolección de información comprendida en los parámetros clínicos se diseñó una encuesta que se envió por correo electrónico a los médicos remitentes para obtener la información clínica pertinente. Aquellos profesionales que no respondieron por vía electrónica fueron contactados por vía telefónica. Análisis estadístico

Para evaluar la asociación entre las variables GA y GI se realizó una prueba de correlación bayesiana (matriz de correlación bayesiana). Para ello, se desarrollaron scripts en lenguaje R (https://cran.r-project.org/) y WinBUGS (https://www.mrc-bsu.cam.ac.uk/software/ bugs/the-bugs-project-winbugs), utilizando la interfaz del lenguaje R, R2WinBUGS (https:// cran.r-project.org/web/packages/R2WinBUGS/ index.html). Se partió de una distribución prior no informativa (prior: anterior a los datos o la muestra; no informativa, otorga la misma probabilidad a cualquier evento y cuyos parámetros a y b equivalen a la unidad, $\mathrm{a}=\mathrm{b}=1$ ) y se corrieron 10000 iteraciones del modelo, descartando las primeras 1000 (Risso \& Risso, 2017).

Para evaluar la asociación entre las variables GA, GI, sexo, edad -informada al remitir la muestra-, ubicación anatómica, edad al momento del diagnóstico (encuesta), existencia de recidivas, tiempo promedio entre recidivas, número de recidivas, tiempo de sobrevida y $\mathrm{PCP}$, la metodología de correlación bayesiana se aplicó mediante el uso de software JASP (https://jaspstats.org/).

Para todos los casos, los datos numéricos discretos, se analizaron usando un modelo transformación a raíz cuadrada, los datos expresados como proporciones se analizaron usando un modelo transformación arcoseno. Para todo el estudio se fijó un nivel de significación $\mathrm{P}<=0.05$.

\section{Resultados}

\section{Estudios histopatológicos}

La distribución de los diferentes tipos morfológicos fue: fibrosarcoma 87,18 \% (8 de ellos presentaron metaplasia osteoide), osteosarcoma extra esquelético 1,71\%, mixosarcoma 5,98\%, sarcoma pleomórfico 3,42 \%, rabdomiosarcoma $1,71 \%$. De los 117 SSI estudiados el 29,91\% presentó GA I, el 64,11 \% GA II y el 5,98 \% GA III . Tres de las recidivas presentaron el mismo GA (GA II) que el tumor primario y una de ellas presentó menor GA (GA I) que aquel (Santelices et al., 2018). Respecto al grado de inflamación, se asignó grado 1 al 15,52 \%, grado 2 al 36,21 \% y grado 3 al $48,27 \%$ de ellos. En este caso, tres de las recidivas presentaron el mismo grado de inflamación que su tumor primario (grado 3 en todos los casos), y una de ellas presentó un grado de inflamación menor (grado 1) que su tumor primario, que era de grado 2 (Santelices et al., 2018). 


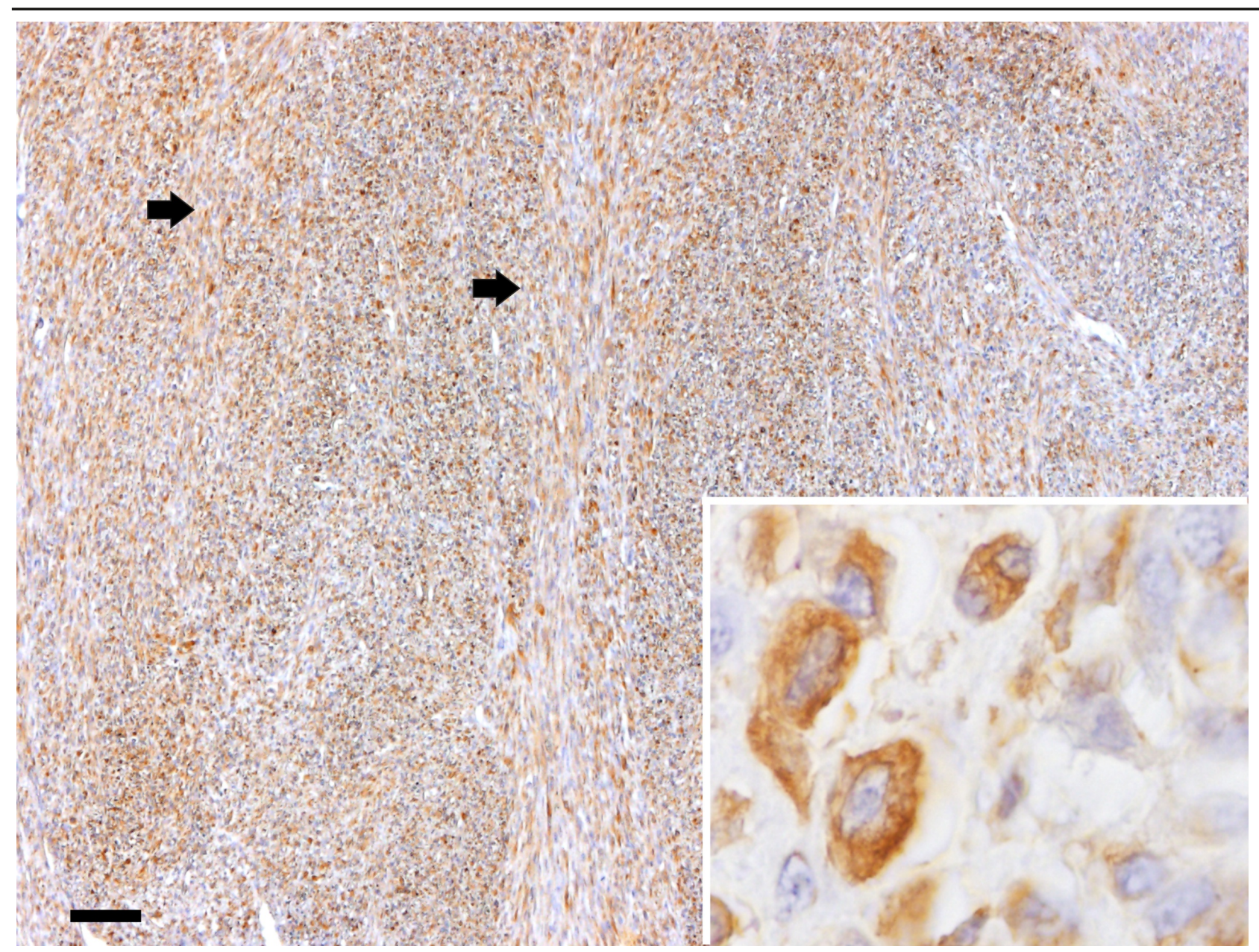

Figura 1. Fibrosarcoma. Patrón característico de haces o fascículos entrelazados de células fusiformes pleomórficas (flechas). Marcación inmunohistoquímica positiva para vimentina. Inserto: detalle magnificado de un área de la imagen principal mostrando la inmunomarcacion citoplasmática. Barra $=200 \mu \mathrm{m}$ (imagen principal); $20 \mu \mathrm{m}$ (inserto). DAB y hematoxilina.

Tabla 1. Clasificación de los sarcomas de acuerdo con los estudios histopatológicos e inmunohistoquímicos.

\begin{tabular}{lllc}
\hline$\%$ & Tipo & Grado de anaplasia & $\%$ \\
\hline 88,89 & Fibrosarcoma (1)a & I & 27,88 \\
& & II & 67,30 \\
& & III & 4,81 \\
1,71 & Osteosarcoma extraesquelético $^{\text {b }}$ & I & 50 \\
& & II & 50 \\
& & III & 0 \\
5,98 & I & 71,43 \\
& Mixosarcoma $^{a}$ & II & 14,28 \\
3,42 & & III & 14,28 \\
& Sarcoma pleomórfico o HFMc & I & 0 \\
& & II & 25 \\
& & III & 75 \\
\hline
\end{tabular}

(1) Ocho de ellos presentaron metaplasia osteoide.

a. Hendrick et al., 1998; b. Sangüesa Nebot et al., 2007; Thompson y Dittmer, 2017; Thompson y Pool, 2002; c. Hendrick, 2017. 


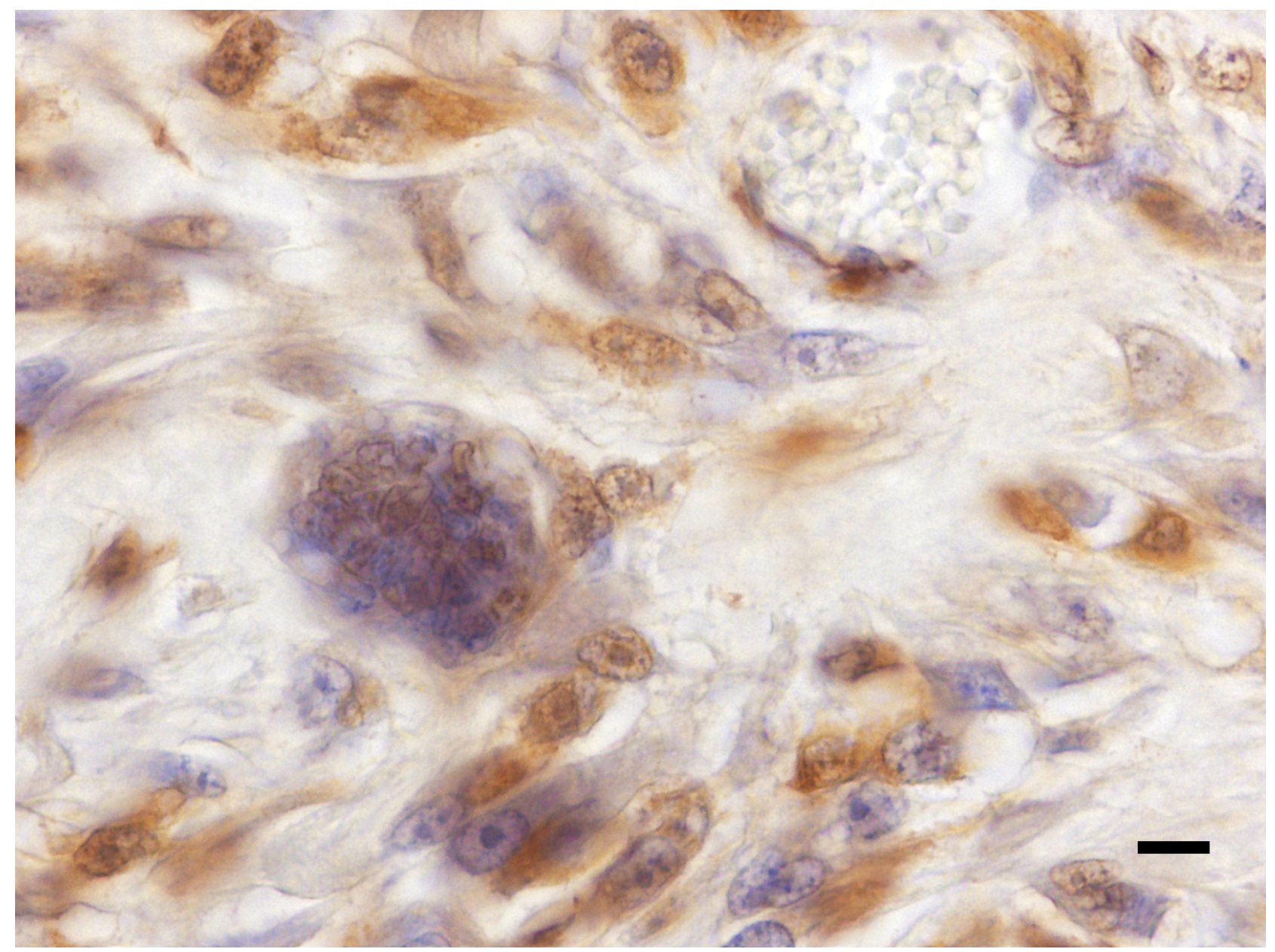

Figura 2. Sarcoma pleomórfico (Hendrick, 2017). Se observa inmunomarcación positiva para alfa-actina. Barra $=200 \mu \mathrm{m}$. DAB y Hematoxilina.

\section{Estudios inmunohistoquímicos}

Todos los sarcomas resultaron negativos para pancitoqueratina y positivos para vimentina (Figura 1). Los 10 sarcomas, seleccionados al azar, en los cuales se evaluó la expresión de desmina resultaron negativos. Los dos SSI que habían sido clasificados preliminarmente como rabdomiosarcomas resultaron negativos para desmina y, por ello, se reclasificaron como fibrosarcomas. Los cuatro clasificados inicialmente como sarcomas pleomórficos (anteriormente denominados HFM) resultaron positivos para $\alpha$-actina de músculo liso (Figura 2) y negativos para desmina, lo que justificó conservar el diagnóstico original.

La clasificación de los SSI de acuerdo con los estudios histopatológicos e inmunohistoquímicos se detallan en la Tabla 1.

El PCP alcanzó un promedio de 50,12 \% de núcleos positivos con puntos extremos de 10,59\% y $89 \%$.

\section{Seguimiento clínico}

Se obtuvieron veinticuatro respuestas efectivas (Tabla 2) que permitieron conocer la evolución de la enfermedad en los casos informados. En relación con la raza de los felinos involucrados 58,12 \% (68 de 117 casos) fueron informados como mestizos o común europeo y $5(4,27 \%)$ fueron siameses; de los $44(37,61 \%)$ restantes no se dispone información. El 46,15 \% (54 de 117) fueron hembras, el 50,43 \% fueron machos y se desconoce el sexo de los restantes 4 animales (3,42\%).

La ubicación anatómica más frecuente fue la parrilla costal (6o animales, 51,28 \%), seguida del flanco $(29,24,79 \%)$. Otros sitios menos frecuentes de aparición fueron la región dorsolumbar (19, 16,24 \%), el cuello (4 casos, $3,42 \%$ ) y la zona interescapular (4 casos, 3,42 \%). Solo se informó un caso de SSI en el muslo $(0,85 \%)$. 
Tabla 2. Información clínica aportada por Médicos Veterinarios remitentes

\begin{tabular}{|c|c|c|c|c|c|}
\hline $\begin{array}{l}\text { Edad al momento } \\
\text { del } \\
\text { diagnóstico } \\
\text { clínico } \\
\text { en años }\end{array}$ & $\begin{array}{l}\text { ¿Recidivó? } \\
\text { Sí/No }\end{array}$ & $\begin{array}{l}\text { Número } \\
\text { de } \\
\text { Recidivas }\end{array}$ & $\begin{array}{l}\text { Tiempo } \\
\text { promedio } \\
\text { entre recidivas } \\
\text { en dias }\end{array}$ & $\begin{array}{l}\text { Sobrevive } \\
\text { Sí/No }\end{array}$ & $\begin{array}{l}\text { Tiempo } \\
\text { de } \\
\text { sobrevida } \\
\text { en dias }\end{array}$ \\
\hline 3,5 & Sí & 2 & 168 & $\mathrm{D}$ & $\mathrm{D}$ \\
\hline 9 & $\mathrm{D}$ & D & $\mathrm{D}$ & D & D \\
\hline 13 & Sí & 1 & 365 & No & 545 \\
\hline 13,5 & Sí & 5 & 342 & No & 1945 \\
\hline 6 & Sí & 2 & 360 & No & 910 \\
\hline 10,5 & Sí & 2 & D & $\mathrm{D}$ & $\mathrm{D}$ \\
\hline 5,5 & $\mathrm{Si}$ & 1 & 360 & No & 545 \\
\hline 9 & $\mathrm{Si}$ & 1 & D & No & 450 \\
\hline 7,67 & $\mathrm{Si}$ & 1 & 180 & No & 635 \\
\hline 15 & $\mathrm{Si}$ & 1 & 540 & No & 1945 \\
\hline 14 & No & 0 & - & No & 120 \\
\hline 9 & $\mathrm{Si}$ & 1 & 274 & No & 635 \\
\hline $\mathrm{D}$ & D & D & D & No & 365 \\
\hline 16 & $\mathrm{D}$ & D & $\mathrm{D}$ & No & 270 \\
\hline 15 & Sí & 3 & 340 & D & D \\
\hline 12,5 & Sí & 1 & 240 & No & D \\
\hline 9 & No & 0 & - & $\mathrm{D}$ & D \\
\hline 7,67 & No & 0 & - & D & D \\
\hline 12 & Sí & 3 & 281,4 & No & 1215 \\
\hline 5 & Sí & 2 & 267 & No & 730 \\
\hline D & Sí & 2 & D & No & $\mathrm{D}$ \\
\hline 8 & Sí & 2 & 135 & No & 270 \\
\hline 6 & Sí & 2 & 255 & No & 665 \\
\hline 12 & Sí & 1 & 180 & No & 365 \\
\hline
\end{tabular}

D: dato desconocido.

Veintidós profesionales informaron la edad del animal al momento del diagnóstico, de estos datos se obtuvo un promedio de 10 años. $\mathrm{Si}$ consideramos la edad informada al momento de remitir la muestra, el promedio de los 103 casos informados es de 8,9 años.

En relación con la ocurrencia de recidivas, de los 24 SSI informados 18 recidivaron (75\%), con un promedio de 1,8 recidivas y variaciones entre 1 y 5 recidivas; 3 no recidivaron $(12,5 \%)$ y de 3 se desconoce si hubo o no recidiva (12,5\%). Se obtuvo información sobre el tiempo entre recidivas en 15 de los 24 casos informados, cuyo promedio fue de 285,83 días con variaciones entre 120 y 540 días. Para ocho casos $(33,34 \%)$ se informó solo una recidiva, en diez casos (41,67 \%) se informó más de una recidiva. Seis de ellos (25\%) recidivaron por primera (o única) vez dentro de los 6 meses, mientras que 4 de ellos recidivaron más de una vez dentro de un período de 2 años (16,67\%).

Respecto al tiempo de sobrevida se obtuvo un promedio de 725,62 días (resultado obtenido a partir de 16 casos en los que se informó este tiempo). Las variaciones oscilaron entre los 120 y 1945 días. En solo tres casos se informó evidencia de metástasis. Uno de ellos presentó masas cutáneas múltiples, otro presentó afección del sistema respiratorio y en el restante se detectó una masa intestinal. Ninguno de estos tumores secundarios cuenta con confirmación histopatológica. La causa de muerte informada fue: eutanasia en 10 casos, deterioro general en 2, insuficiencia renal en $3 \mathrm{y}$ posible metástasis en sistema respiratorio en 1. En 8 casos se desconoce la causa de la muerte.

Solo dos de los Médicos Veterinarios informaron haber realizado cirugía radical. De ellos, solo uno cuenta con información sobre la evolución del paciente. Este último, informa que el paciente sufrió una recidiva y luego de un tratamiento quirúrgico agresivo en el que se extirpó, junto con la masa, pared costal y abdominal, permaneció libre de tumor hasta su muerte por insuficiencia renal. El tiempo de sobrevida en este caso fue de 1945 días.

Cuando se consultó a los profesionales por la posibilidad de vacunación en el sitio de aparición de la neoplasia en cuestión, 5 respondieron que con certeza recibieron la aplicación de vacunas en ese sitio, 15 que existía la posibilidad de vacunación en el sitio. Cuando se los consultó sobre la vacunación antirrábica (específicamente), 4 manifestaron certeza de vacunación antirrábica en el sitio y 16 que existía esa posibilidad. De los restantes casos, 4 en total, los médicos actuantes no pudieron informar sobre antecedentes de vacunación, argumentando que el caso era atendido por otros colegas previamente al diagnóstico del tumor y que recibieron el caso por derivación. 
Tabla 3. Matriz de correlación Bayesiana (https://jasp-stats.org/)

\begin{tabular}{|c|c|c|c|}
\hline Variables & & Pearson cc & p-value ${ }^{(1)}$ \\
\hline GA & Sexo & $-0,098$ & 0,303 \\
\hline GA & Raza & $\mathrm{NaNa}^{(2)}$ & ------- \\
\hline GA & Edad & 0,03 & 0,76 \\
\hline GA & UA & $-0,076$ & 0,413 \\
\hline GA & EMD & 0,111 & 0,623 \\
\hline GA & TPR & $-0,124$ & 0,661 \\
\hline GA & NR & $-0,296$ & 0,1930 \\
\hline GA & Tsd & $-0,351$ & 0,1830 \\
\hline GA & Rsn & $-0,04$ & 0,8630 \\
\hline GI & Sexo & $-0,129$ & 0,1720 \\
\hline GI & Edad & 0,033 & 0,7370 \\
\hline GI & Raza & $\mathrm{NaNa}^{a}(2)$ & --.--- \\
\hline GI & UA & $-0,106$ & 0,2560 \\
\hline GI & EMD & $-0,324$ & 0,7310 \\
\hline GI & TPR & $-0,151$ & 0,5920 \\
\hline GI & NR & $-0,03$ & 0,8960 \\
\hline GI & Tsd & $-0,3$ & 0,2590 \\
\hline GI & Rsn & 0,198 & 0,3900 \\
\hline PCP & Sexo & 0,04 & 0,387 \\
\hline PCP & Raza & $\mathrm{NaN}^{\mathrm{a}(2)}$ & ----- \\
\hline PCP & Edad & $-0,341$ & 0,01 \\
\hline PCP & UA & 0,039 & 0,39 \\
\hline PCP & EMD & $-0,442$ & 0,02 \\
\hline PCP & TPR & $-0,124$ & 0,661 \\
\hline PCP & NR & $-0,193$ & 0,246 \\
\hline PCP & Tsd & $-0,072$ & 0,395 \\
\hline PCP & Rsn & $-0,072$ & 0,481 \\
\hline PCP & GA & 0,244 & 0,037 \\
\hline PCP & GI & $-0,01$ & 0,528 \\
\hline EMD & TPR & 0,451 & 0,046 \\
\hline EMD & Rsn & $-0,065$ & 0,607 \\
\hline EMD & NR & 0,122 & 0,305 \\
\hline EMD & Tsd & 0,23 & 0,205 \\
\hline TPR & Rsn & $\mathrm{NaN}^{\mathrm{a}(2)}$ & -.---.- \\
\hline TPR & NR & 0,049 & 0,431 \\
\hline TPR & Tsd & 0,696 & 0,006 \\
\hline Rsn & NR & 0,564 & 0,004 \\
\hline Rsn & Tsd & 0,342 & 0,116 \\
\hline NR & Tsd & 0,635 & 0,007 \\
\hline
\end{tabular}

(1) $\mathrm{P}>0.05$ = no hay correlación significativa. (2) $\mathrm{NaN}^{\mathrm{a}}=$ Varianza $=0$, no se realiza el análisis. Pearson cc = coeficiente de correlación; p-value = valor de $\mathrm{P} ; \mathrm{GA}=$ grado de anaplasia; $\mathrm{UA}=$ ubicación anatómica; Edad: edad informada al momento de la remisión de la muestra en años; EMD = edad al momento del diagnóstico clínico en años; TPR = tiempo promedio entre recidivas en días; NR = número de recidivas; Tsd = tiempo de sobrevida en días; $\mathrm{Rsn}$ = recidiva, $\mathrm{si}$ - no; GI= grado de inflamación; PCP= porcentaje de células proliferantes.

\section{Análisis estadístico}

No hubo asociación estadísticamente significativa entre las variables GA y GI $(\mathrm{P}=0.203)$. Tampoco hubo asociación entre las variables GA y GI con sexo, edad (al momento de remisión de la muestra), ubicación anatómica, edad al momento del diagnóstico (informado en encuesta), existencia de recidivas, tiempo promedio entre recidivas en días, número de recidivas y tiempo de sobrevida. Del mismo modo, no se halló asociación entre el PCP y, sexo, ubicación anatómica, GI, existencia de recidivas, tiempo promedio entre recidivas y tiempo de sobrevida (Tabla 3 ).

Se halló asociación estadísticamente significativa entre el PCP y las siguientes variables: GA
( $\mathrm{P}=0,037)$, edad al momento del diagnóstico $(\mathrm{P}=0,02)$, edad informada al momento de remisión de la muestra $(\mathrm{P}=0,01)$ y número de recidivas $(\mathrm{P}=0,026)$ (Tabla 3$)$.

También, se demostró correlación significativa directa entre: edad al momento del diagnóstico y tiempo promedio entre recidivas $(\mathrm{P}=0,046)$, tiempo promedio entre recidivas $\mathrm{y}$ tiempo de sobrevida $(\mathrm{P}=0,006)$, número de recidivas y tiempo de sobrevida $(\mathrm{P}=0,007)$ (Tabla 3).

No se evaluó la asociación entre el PCP y raza debido a que todos los animales de los que se conoció la raza y a los que se realizó inmunomarcación con anticuerpo anti-PCNA eran gatos mestizos; 5 profesionales no informaron ese dato. 


\section{Discusión y conclusiones}

El fibrosarcoma es el SSI más frecuente en la República Argentina, aunque también se encuentran, en menor proporción, otros tumores como: sarcomas pleomórficos (HFM), osteosarcomas extra esqueléticos y mixosarcomas. Estos hallazgos son similares a los descriptos en otros países en los que se realizaron estudios epidemiológicos, tales como EE.UU., España, Italia, Canadá (Hendrick, 1999; Martano et al., 2011; Nieto, 2003; Wilcock et al., 2012).

Según Hendrick (2017), los sarcomas pleomórficos o HFM poseen un fenotipo fibroblástico/miofibroblástico: positivo a vimentina, y con positividad variable para actina y desmina. En este estudio, los resultados inmunohistoquímicos de estos SSI (positivos para vimentina y a-actina de músculo liso, y negativos para desmina) fueron compatibles con el fenotipo descripto por Hendrick (2017), por este motivo se confirmó su diagnóstico.

En relación con el grado de anaplasia de los SSI, al igual que lo informado por Couto (2002) y Nieto (2003), las neoplasias de GA II fueron las más numerosas. Tres de las recidivas presentaron el mismo GA (DA II) que el tumor primario y una de ellas presentó un GA menor (GA I).

Con respecto al GI, la mayoría de los sarcomas fueron de grado 3. En este caso, tres de las recidivas presentaron el mismo GI que el tumor primario, en todos los casos GI 3, y una de ellas tuvo un GI menor (grado 1) que el tumor primario que presentó GI 2. Esta información concuerda con la presentada por Couto (2002), que manifiesta que la mayoría de las neoplasias presentan abundante inflamación, tanto en los tumores primarios como en las recidivas. No se halló asociación entre GA y GI, tal como ocurrió en trabajos previos (Couto, 2002).

El promedio de edad al momento del diagnóstico fue mayor, tanto en las encuestas (10 años), como en los protocolos de remisión de muestras (8,9 años), al informado por otros autores (8,1 años) (Goldschmidt \& Hendrick, 2002; Hendrick et al., 1994). Incluso, el obtenido de las encuestas, es mayor que el informado para los sarcomas no asociados a sitios de inoculación (9,2 años) (Goldschmidt \& Hendrick, 2002; Hendrick et al., 1994). Esto podría deberse a que la consulta en nuestro medio se retrasa en comparación con lo que ocurre en países del primer mundo.

Los sarcomas estudiados presentaron alta recurrencia. Esto concuerda con lo comunicado por Hendrick (1999). Sin embargo, solo un tercio lo hizo dentro de los seis meses, período en el que este autor ubica la mayoría de las primeras recidivas y solo el 16,67\% de los casos presentaron más de una recidiva en un período de dos años, porcentaje muy inferior al mencionado por este autor (22 \%) (Hendrick et al., 1999). Es necesario considerar que solo 5 (20,84\% de los 24 casos con información sobre seguimiento clínico, y el $31 \%$ de los 16 con información sobre tiempo de sobrevida) alcanzaron los dos años de sobrevida (730 días). Al igual que informaron otros autores (Martano, 2011) no se encontró asociación entre el GA y la ocurrencia de recidivas.

Según los resultados estadísticos obtenidos, los animales de mayor edad (al momento del diagnóstico) presentaron intervalos mayores entre recidivas y los animales cuyo período entre recidivas era más extenso tenían mayor expectativa de sobrevida. Sin embargo, a mayor tiempo de sobrevida mayor es el número de recidivas informadas. No hallamos en la bibliografía consultada ninguna información previa que vincule estas variables.

Respecto a la relación que el tipo de tratamiento mantiene con la sobrevida y con la ocurrencia de recidivas contamos con escasa información. El único caso en el cual se informa la realización de una cirugía radical no presentó recidivas luego de esta y fue uno de los dos pacientes con sobrevida más extensa, hechos que coinciden con lo hallado por otros autores (Hendrick, 1999). Sin embargo, es imposible extraer conclusiones definitivas al respecto, ya que se trata de un único caso.

La variable PCP se asoció significativamente con el GA, la edad al momento del diagnóstico (relación inversa) y el número de recidivas (relación inversa). Esto pareciera indicar que cuanto menor es la edad (al momento del diagnóstico y de remisión de la muestra), mayor es el PCP y cuanto mayor la capacidad proliferativa de estas neoplasias las posibilidades de recidivar son menores. Sin embargo, si bien existen antecedentes de neoplasias que se comportan más agresivamente en jóvenes (Poles et al., 2016), es indispensable disponer de más información sobre otras variables relacionadas. Otras variables, tales como el tamaño de la neoplasia al momento del diagnóstico y la agresividad del tratamiento quirúrgico realizado, no están disponibles y serían importantes para evaluar, en forma más profunda, la significación biológica de estos resultados estadísticos.

El presente trabajo representa el primer estudio epidemiológico sobre los SSI en Argentina. La mayoría de los aspectos analizados, tales como diagnósticos histopatológicos, la presencia de inflamación, el GA, los sitios de aparición y la ausencia de predisposición sexual, son similares a los hallados en otros países. La mayor edad al momento del diagnóstico podría deberse a diferencias en el momento en que los propietarios de los gatos realizan la consulta con el médico veterinario. 


\section{Agradecimientos}

A Fabiana Confente y Melina Díaz por su invaluable labor en el área técnica. El presente trabajo contó con el siguiente financiamiento: Proyectos de la Universidad Nacional de La Plata (11/V229, 11/V232, S/N).

\section{Conflicto de intereses}

No existe conflicto de intereses, incluyendo entre estos últimos las relaciones financieras, personales o de otro tipo con otras personas $\mathrm{u}$ organizaciones que pudieran influir de manera inapropiada en el trabajo

\section{Bibliografía}

Briscoe CM, Lipscomb TP, McKinney L. 1998. Pulmonary metastasis of a feline vaccination-site fibrosarcoma. Journal of Veterinary Diagnostic Investigation. 10(1):79-82.

doi: 10.1177/104063879801000114

Burton G, Mason KV. 1997. Do postvaccinal sarcomas occur in Australian cats? Australian Veterinary Journal. 75(2):102-8.

doi: 10.1111/j.1751-0813.1997.tb14167.x

Chang HW, Ho SY, Lo HF, Tu YC, Jeng CR, Liu CH, Wang FI, Pang VF. 2006. Vaccine-associated Rhabdomyosarcoma with Spinal Epidural Invasion and Pulmonary Metastasis in a Cat. Veterinary Pathology. 43(1): 55-58.

doi: $10.1354 / v p .43-1-55$

Couto SS, Griffey SM, Duarte PC, Madewell BR. 2002. Feline vaccine-associated fibrosarcoma: morphologic distinctions. Veterinary Pathology. 39(1):33-41. doi: 10.1354/vp.39-1-33

Davidson EB, Gregory CR, Kass PH. 1997. Surgical excision of soft tissue fibrosarcomas in cats. Veterinary Surgery. 26(4): 265-9.

doi: 10.1111/j.1532-950X.1997.tbo1497.x

Deim Z, Pálmai N, Cserni G. 2008. Feline vaccineassociated fibrosarcoma induced by aluminium compound in two cats: short communication. Acta Veterinaria Hungarica. 56(1):111-6.

doi: 10.1556/AVet.56.2008.1.11.

Dubielzig RR, Hawkins KL, Miller PE. 1993. Myofibroblastic sarcoma originating at the site of rabies vaccination in a cat. Journal of Veterinary
Diagnostic Investigation. 5(4):637-8.

doi: 10.1177/104063879300500427

Goldschmidt MH, Hendrick MJ. Tumors of the Skin and Soft Tissues. En: Meuten DJ 2002. Tumors in Domestic Animals. 4th Edition. Iowa, Ed Blackwell Publishing, pp. 45-117.

Hendrick MJ, Shofer FS, Goldschmidt MH, Haviland JC, Schelling SH, Engler SJ, Gliatto JM. 1994. Comparison of fibrosarcomas that developed at vaccination sites and at nonvaccination sites in cats: 239 cases (1991-1992). Journal of the American Veterinary Medical Association. 205(10): 1425-9.

Hendrick MJ, Brooks JJ. 1994. Postvaccinal sarcomas in the cat: histology and immunohistochemistry. Veterinary Pathology. 31(1):126-9. doi: 10.1177/030098589403100121

Hendrick MJ, Goldschmidt MH. 1991. Do injection site reactions induce fibrosarcomas in cats? Journal of the American Veterinary Medical Association. 5(8): 968.

Hendrick MJ, Goldschmidt MH, ShoferFS, Wang YY, Somlyo AP. 1992. Postvaccinal sarcomas in the cat: epidemiology and electron probe microanalytical identification of aluminum. Cancer Research. 52(19):5391-4.

Hendrick MJ, Mahaffey EA, Moore FM, Vos JH, Walder EJ. Histological classification of mesenchymal tumors of skin and soft tissues of domestic animals. En: 1998. World Health Organization International Classification of Tumors in Domestic Animals. 2nd Edition, Washington DC, Ed. Armed Forces Institute of Pathology, pp. 15-60.

Hendrick MJ. 1999. Feline vaccine-associated sarcomas. Cancer Investigation. 17(4):273-7.

Hendrick, MJ. Mesenchymal Tumors of the Skin and Soft Tissues. En: Meuten DJ. 2017. Tumors in Domestic Animals, 5ta Edición, Iowa, Ed. Wiley Blackwell, pp. 142-75.

Hershey AE, Sorenmo KU, Hendrick MJ, Shofer FS, Vail DM. 2000. Prognosis for presumed feline vaccine-associated sarcoma after excision: 61 cases (1986-1996). Journal of the American Veterinary Medical Association. 216(1):58-61.

doi: 10.246o/javma.2000.216.58

Kass PH, Barnes WG Jr, Spangler WL, Chomel BB, Culbertson MR. 1993. Epidemiologic evidence for a causal relation between vaccination and fibrosarcoma tumorigenesis in cats. Journal of the 
American Veterinary Medical Association. 203(3): 396-405.

MacEwen EG. Powers BE, Macy D, Withrow SJ. Soft Tissue Sarcomas. En: Withrow SJ, MacEwen EG. 2001. Small Animal Clinical Oncology. 3th Edition. Philadelphia, W.B. Ed Saunders Company, pp. 283-304.

Madewell BR, Griffey SM, McEntee MC, Leppert VJ, Munn RJ. 2001. Feline Vaccine-associated Fibrosarcoma: An Ultrastructural Study of 20 tumors (1996-1999). Veterinary Pathology. 38(2): 196-202. doi: 10.1354/vp.38-2-196

Martano M, Morello E, Buracco P. 2011. Feline injection-site sarcoma: past, present and future perspectives. Veterinary Journal. 188(2):136-41. doi: 10.1016/j.tvjl.2010.04.025

Nieto A, Sánchez MA, Martínez E, Rollán E. 2003. Immunohistochemical expression of p53, Fibroblast Growth Factor-b, and Transforming Growth Factor- $\alpha$ in feline vaccine-associated sarcomas. Veterinary Pathology. 40(6):651-8.

doi: 10.1354/vp.40-6-651

O’Byrne KJ, Dalgleish AG. 2001. Chronic immune activation and inflammation as the cause of malignancy. British Journal of Cancer. 85(4):47383. doi: 10.1054/bjoc.2001.1943

Poles GC, Clark DE, Mayo SW, Beierle EA, Goldfarb M, Gow KW, Goldin A, Doski JJ, Nuchtern JG, Vasudevan SA, Langer M. 2016. Colorectal carcinoma in pediatric patients: A comparison with adult tumors, treatment and outcomes from the National Cancer Database. Journal of pediatric surgery. 51(7):1061-6.

doi: 10.1016/j.jpedsurg.2015.11.005

Risso MA, Risso P. Capítulo I. El teorema de Bayes. En: Risso MA, Risso P. 2017. Una Introducción a la Estadística Bayesiana: Uso de Lenguaje R y WinBUGS, 1raEdición, La Plata, Ed. Vuelta a casa, pp. 19-38.

Robledo Martín J. 2005. Diseño de muestreo (II). Nure Investigación. [En línea] Disponible en: http://www.nureinvestigacion.es/OJS/index.php/ nure/article/viewFile/214/199

Consultado: 02/04/2019
Rudmann DG, Van Alstine WG, Doddy F, Sandusky GE, Barkdull T, Janovitz EB. 1996. Pulmonary and mediastinal metastases of a vaccination-site sarcoma in a cat. Veterinary Pathology. 33(4):466-9.

doi: 10.1177/030098589603300422

Sandler I, Teeger M, Best S. 1997. Metastatic vaccine associated fibrosarcoma in a 10-year-old cat. The Canadian Veterinary Journal. 38(6):374.

Sangüesa Nebot MJ, Cabanes Soriano F, Valverde Mordt C. 2007. Osteosarcoma extraesquelético. Caso clínico. Revista Española De Cirugía Osteoarticular. 42:88-92.

Santelices Iglesias OA, Wright C, Duchene AG, Risso MA, Risso P, Zanuzzi CN, Nishida F, Lavid A, Confente F, Díaz M, Portiansky EL, Gimeno EJ, Barbeito CG. 2018. Association between degree of anaplasia and degree of inflammation with the expression of Cox-2 in feline injection site sarcomas. Journal of Comparative Pathology. 165:45-51. doi: 10.1016/j.jcpa.2018.09.002

Shaw SC, Kent MS, Gordon IK, Collins CJ, Greasby TA, Beckett LA, Hammond GM, Skorupski KA. 2009. Temporal changes in characteristics of injection-site sarcomas in cats: 392 cases (1990-2006). Journal of the American Veterinary Medical Association. 234(3):376-80. doi: 10.2460/javma.234.3.376

Thompson KG, Pool RR. Tumors of Bones. En: Meuten DJ. 2002. Tumors in Domestic Animals, 4ta Edición, Iowa, Ed. Blackwell Publishing, pp. 245-317.

Thompson KG, Dittmer KE. Tumors of Bone. En: Meuten DJ. 2017. Tumors in Domestic Animals, 5ta Edición, Iowa, Ed. Wiley Blackwell, pp. 356424.

Wilcock B, Wilcock A, Bottoms K. 2012. Feline postvaccinal sarcoma: 20 years later. The Canadian veterinary journal. 53(4):430-4.

Woodward KN. 2011. Origins of Injection-Site Sarcomas in Cats: The possible role of chronic inflammation-a review. International Scholarly Research Network Veterinary Science. 2011:1-16. doi: 10.5402/2011/210982 